Data and Text Mining

\title{
dom2vec: Unsupervised protein domain embeddings capture domains structure and function providing data-driven insights into collocations in domain architectures
}

\author{
Damianos P. Melidis ${ }^{1, *}$, Brandon Malone ${ }^{2}$, Wolfgang NejdI ${ }^{1,3}$ \\ ${ }^{1}$ L3S Research Center, Leibniz University of Hannover, Hanover, 30167, Germany, \\ ${ }^{2}$ NEC Laboratories Europe, Heidelberg, 69115, Germany and \\ ${ }^{3}$ Knowledge-based Systems Laboratory, Leibniz University of Hannover, Hanover, 30167, Germany. \\ * To whom correspondence should be addressed. \\ Associate Editor: XXXXXXX \\ Received on XXXXX; revised on XXXXX; accepted on XXXXX
}

\begin{abstract}
Motivation: Word embedding approaches have revolutionized Natural Language Processing NLP research. These approaches aim to map words to a low-dimensional vector space in which words with similar linguistic features are close in the vector space. These NLP approaches also preserve local linguistic features, such as analogy. Embedding-based approaches have also been developed for proteins. To date, such approaches treat amino acids as words, and proteins are treated as sentences of amino acids. These approaches have been evaluated either qualitatively, via visual inspection of the embedding space, or extrinsically, via performance on a downstream task. However, it is difficult to directly assess the intrinsic quality of the learned embeddings.

Results: In this paper, we introduce dom2vec, an approach for learning protein domain embeddings. We also present four intrinsic evaluation strategies which directly assess the quality of protein domain embeddings. We leverage the hierarchy relationship of InterPro domains, known secondary structure classes, Enzyme Commission class information, and Gene Ontology annotations in these assessments. These evaluations allow us to assess the quality of learned embeddings independently of a particular downstream task. Importantly, allow us to draw an analog between the local linguistic features in nature languages and the domain structure and function information in domain architectures, thus providing datadriven insights into the context found in the language of domain architectures. We also show that dom2vec embeddings outperform, or are comparable with, state-of-the-art approaches on downstream tasks.

Availability: The protein domain embeddings vectors and the entire code to reproduce the results are available at https://github.com/damianosmel/dom2vec.

Contact: melidis@|3s.uni-hannover.de
\end{abstract}

\section{Introduction}

A primary way of how proteins evolve is through rearrangement of their functional/structural units, known as protein domains (Moore et al., 2008; Forslund and Sonnhammer, 2012). The domains are independent folding and functional modules and so they exhibit conserved sequence segments. Prediction algorithms exploited this information and used as input features the domain composition of a protein for various tasks. For example (Chou and Cai, 2002) classified the cellular location and (Forslund and Sonnhammer, 2008; Doğan et al., 2016) predicted the associated Gene

(c) The Author 2020
Ontology (GO) terms. There exist two ways to represent domains; either by the linear order in a protein, domain architecture, (Scaiewicz and Levitt, 2015), or by a graph where nodes are domains and edges connect domain that co-exist in a protein (Moore et al., 2008; Forslund and Sonnhammer, 2012).

Moreover, (Yu et al., 2019) investigated if the domains architecture has a grammar as a natural spoken language. They compared the bigram entropy of domain architecture for PFAM domains (Sonnhamme et al., 1998) to the respective entropy of the English language, showing that although it was lower than the English language, it was significantly different from a language produced after shuffling the domains. Prior to this result, methods had exploited domain architecture representation to 
various applications, such as fast homology search (Terrapon et al., 2013) and retrieval of similar proteins (Marchler-Bauer et al., 2016).

Word embeddings are unsupervised learning methods which have as input large corpora and they output a dense vector representation of words contained in the sentences of these documents based on the distributional semantic hypothesis, that is the meaning of a word can be understood by its context. Thus a word vector represents local linguistic features, such as lexical or semantical information, of the respective word. Several methods to train word embeddings have been established, for example (Collobert and Weston, 2008; Mikolov et al., 2013b; Pennington et al., 2014). These representations have been shown to hold several properties such as analogy and grouping of semantically similar words (Mikolov et al., 2013a; Drozd et al., 2016). Importantly, these properties are learned without the need of a labeled data set. Word embeddings are currently the mainstream input for neural networks in the Natural Language Processing (NLP) field as first they reduce the feature space (compared to 1-hot representation) and second they provide word features that encapsulate relation between words based on linguistic features. The use of word embeddings improved the performance on most of the tasks (sentiment analysis, Named Entity Recognition (NER), etc).

Various methods to create embeddings for proteins are proposed (Asgari and Mofrad, 2015; Yang et al., 2018; Bepler and Berger, 2019; Asgari et al., 2019; Heinzinger et al., 2019; Alley et al., 2019). ProtVec fragmented the protein sequence in 3-mers for all possible starting shifts, then learned embeddings for each 3-mer and represented the respective protein as the average of its constituting 3-mer vectors (Asgari and Mofrad, 2015). SeqVec utilized and extended the Embeddings from Language Models (ELMo) (Peters et al., 2018) to learn a dense representation per amino acid residue, resulting in matrix representations of proteins, created by concatenating their learned residue vectors (Heinzinger et al., 2019).

Both approaches evaluated the learned representations qualitatively and quantitatively. Qualitatively by having plotted the reduced 2-D embedding space and reported the appearance of cluster of protein with similar properties (biophysical/chemical, structural, enzymatic, taxonomic). For quantitative evaluation, they measured the improvement of performance in down-stream tasks (protein family classification, cellular location prediction, etc).

Focusing on their qualitative evaluation, it lacks the ability to assess different learned embeddings in a sophisticated manner. Indeed for word embeddings there is an increase in methods to evaluate words representations intrinsically such as (Schnabel et al., 2015). All these techniques are thoroughly summarized by the survey (Lastra-Díaz et al., 2019). This is a fruitful direction, because having such evaluation metrics we can validate the knowledge acquired by all trained embedding spaces. And further we can select the best performing space to input to the machine learning algorithm. However creating embeddings for single residues or $k$-mers residues does not enable intrinsic evaluation methods, as biological information for each such fine-grained feature cannot be obtained for all disposed proteins (that embeddings are learned from).

Given that InterPro database (release 70.0) contains domains for 80.9\% of the UniProtKnowledgeBase (UniProtKB) sequences (The UniProt Consortium, 2017) and includes associated biological knowledge for the majority of domains (Mitchell and et al., 2019), we trained domain embeddings and evaluated them both in intrinsic and extrinsic way.

This work has five major contributions:

- We constructed the domain architectures of all UniProtKB sequences with an identified InterPro domain. Then using word2vec method, we learned the vector representation for each InterPro domain based on the constructed domain architectures.

- We established intrinsic evaluation methods based on four distinct biological information for a domain. First we evaluated the learned embedding space by domain hierarchy (tree structure capturing family-subfamily relations provided by InterPro). We investigated the performance of a nearest neighbor classifier, $C_{\text {nearest }}^{d}$, to predict the secondary structure class provided by SCOPe (Fox et al., 2013) and the primary EC class in the learned embedding space. We examined the performance of $C_{\text {nearest }}^{d}$ classifier to predict the GO molecular function class for three example model organisms and one human pathogen.

- As a by-product of the intrinsic evaluation, we observed that $C_{\text {neares }}^{d}$ reaches adequate accuracy, compared to $C_{\text {nearest }}^{d}$ on randomized domains vectors, for secondary structure, enzymatic and GO molecular function. Thus we hypothesized the analog between word embedding clustering by local linguistic features and protein domains clustering by domains structure and function, which in turn, brings data-driven insights into the semantic context of domain architectures.

- To evaluate our embeddings extrinsically, we inputted the learned domains embeddings to simple neural networks and compared their performance with state-of-the-art methods in three full-protein tasks; surpassing both state-of-the-art methods for the two tasks, EC primary class and toxin presence, and being comparable in the cellular location prediction task.

- We make available the trained domains embeddings to be used for protein supervised learning tasks by the research community.

\section{Related work}

\subsection{Protein embedding methods}

Works on protein embeddings can be divided in two methods based on their word assignment, the former learn embeddings for constant amino acid subsequences and the latter for variable length subsequences.

\subsubsection{Constant length subsequence vectors}

Early work in protein embeddings (Asgari and Mofrad, 2015) (ProtVec) belongs to this category. In this work, the authors split the protein sequence in 3-mers with the 3 possible starting shifts. They then used the skip-gram model from (Mikolov et al., 2013b) to learn the embeddings for each distinct 3-mer. The embedding for a protein was taken as the mean of all its 3-mers. (Yang et al., 2018) adapted ProtVec by using doc2vec (Le and Mikolov, 2014) to find the whole protein embedding. Similarly (Woloszynek et al., 2019) computed embeddings for all $k$-mers using a range of $k$; they also used doc2vec to find the whole protein embedding. (Bepler and Berger, 2019) learned an embedding of each amino acid position incorporating global structural similarity between a pair of proteins and contact map information for each protein. They represented the alignment of two proteins as a soft symmetric alignment of the embeddings of the protein residues. Recently, SeqVec (Heinzinger et al., 2019) applied and extended ELMo model (Peters et al., 2018) to learn a contextualized embedding per amino acid position. Recently, UniRep was introduced (Alley et al., 2019) to learn embeddings for amino acids by a language model that uses RNNs.

\subsubsection{Variable-length subsequence vectors}

ProtVecX (Asgari et al., 2019) embeds proteins by first extracting (variable-length) motifs from proteins using a data compression algorithm, byte-pair encoding (Gage, 1994). ProtVec is then used to learn embeddings for the motifs.

\subsection{Extrinsic evaluation methods}

Extrinsic evaluation methods, like performance on a supervised learning task, are most commonly used to evaluate the quality of embeddings. To 
date, different papers have evaluated performance on different downstream tasks and using different datasets.

Sequence-level prediction (Asgari and Mofrad, 2015) predicted protein family and disorder, while (Yang et al., 2018) evaluated embeddings by predicting the Channelrhodopsin (ChR) localization, the thermostability of Cytochrome P450, the Rhodopsin absorption wavelength and the Epoxide hydrolase enantioselectivity. (Bepler and Berger, 2019) predicted transmembrane regions of proteins, and (Heinzinger et al., 2019) used subcellular localization and water solubility as validation. (Asgari et al., 2019) consider venom toxin, subcellular localization, and enzyme primary class predictions tasks for downstream validation. (Alley et al., 2019) predicted stability of proteins and the phenotype of diverse variants of the green fluorescent protein.

Per-residue prediction (Heinzinger et al., 2019) predicted secondary structure and intrinsic disorder and (Alley et al., 2019) predicted functional effect of single amino acid mutations.

\subsection{Qualitative evaluation methods}

Many of the described approaches use some form of qualitative evaluation. Commonly, the learned embeddings are projected to two dimensions using techniques like tSNE (Maaten and Hinton, 2008) or UMAP (McInnes et al., 2018) to visually inspect the embeddings. While such approaches are helpful to gain trust in the embeddings, they do not provide a rigorous approach to compare the quality of multiple embedding spaces, such as those found when using different hyperparameters for the embedding models.

(Asgari and Mofrad, 2015) used a qualitative intrinsic evaluation approach for ProtVec. They visualized the 2-dimensional reduced protein space along with their biophysical and biochemical properties to report the appearance of clusters with similar biochemical properties.

For intrinsic evaluation they (Heinzinger et al., 2019) visualized the 2-dimensional reduced protein space along with secondary structure class, the EC and the taxonomic kingdoms per protein.

\section{Materials and methods}

Our approach is summarized in Figure 1, in the following we explain the methodology for each part of the approach.

\section{1 dom2vec}

We now describe dom2vec, our novel approach for learning protein domain embeddings based on domain architectures.

\subsubsection{Building domain architectur}

Hereafter we will refer to the sequence of domains in a protein as its domain architecture. We consider two distinct strategies to represent a protein based on its domain architecture: non-overlapping and nonredundant. In both cases, the sequences are based on protein domain entries from Interpro. For efficiency, an interval tree is built for each protein to detect overlapping domains, and each protein is split into regions with overlapping domains.

Non-overlapping sequences. For each region with overlapping domains, all domains except the longest are removed.

Non-redundant sequences. For each region with overlapping domains, all domains with the same Interpro identifier, except the longest domain with each identifier, are removed.

In both cases, the domains in each protein are sorted by start position to construct its domain architecture. Following the approach of (Doğan et al., 2016) we also added the "GAP" domain to annotate more than 30 amino acid subsequence that does not match any InterPro domain entry.

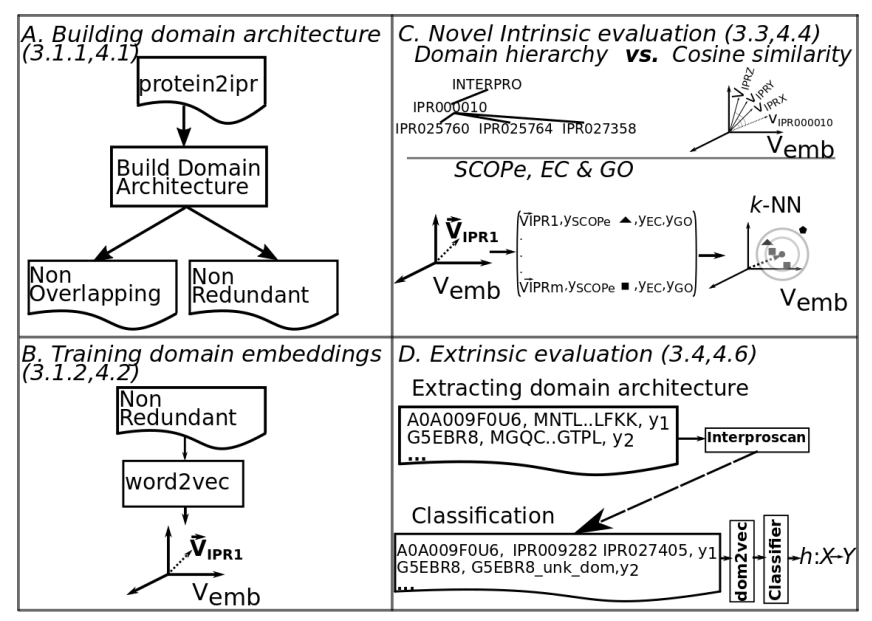

Fig. 1. Summary of our approach divided in four parts, building two forms of domain architecture, training domain embeddings, performing intrinsic and extrinsic evaluation of embeddings. Numbers in parentheses indicate the sections discussing the respective part.

\subsubsection{Training domain embeddings}

Next, we learned task-independent embeddings for each domain using two variants of word2vec (Mikolov et al., 2013b): continuous bag of words CBOW and SKIP. See (Mikolov et al., 2013b) for technical details on the difference between these approaches. In our context, each domain is taken as a word, and each protein sequence is considered as a sentence. Thus, after learning, each domain is associated with a task-independent embedding.

In Section 3.3, we consider several intrinsic evaluation strategies to determine the quality of these embeddings for various hyperparamers of word 2 vec.

\subsection{Qualitative evaluation}

As a preliminary evaluation strategy, we used qualitative evaluation approaches adopted in existing work. To follow the qualitative approach of ProtVec and SeqVec we also visualized the embedding space for selected domain superfamilies, to answer the following research question,

RQ: Did vectors of each domain superfamily form a cluster in the $V_{e m b}$ ?

Evaluation To find out, we added the vector of each domain in a randomly chosen domain superfamily to an empty space. Then we performed principle component analysis (PCA) (Pearson, 1901) to reduce the space in two dimensions and observed the formed clusters.

\subsection{Novel intrinsic evaluation methods}

Previous work has evaluated the quality of embeddings only indirectly by measuring performance on downstream, supervised tasks, as described in Section 3.4. However, in the natural language processing field, the quality of a learned word embedding space is often evaluated intrinsically by considering relationships among words, such as analogies. Such an evaluation is important because it ensures the learned embeddings are meaningful without choosing a specific downstream task.

Such intrinsic evaluations cannot be performed for the ProtVec and SeqVec protein embeddings, because in contrast with these approaches dom 2 vec directly learns embeddings for protein domains. That is, protein domains are typically represented using structures like profile hidden Markov models (HMM) (Eddy, 1998) rather than unique amino acid sequences. Thus it is not straightforward how sequence feature embeddings 
can be combined for a given profile HMM in order to evaluate the learned sequence embeddings in the way that we will describe next.

Thus, prior approaches, such as ProtVec, SeqVec, cannot learn unique embeddings per domain not allowing us to perform the same evaluation tasks for the existing protein embeddings.

We propose four intrinsic evaluation approaches for domain embeddings: domain hierarchy based on the family/subfamily relation, secondary structure class, Enzymatic Commission (EC) primary class, and Gene Ontology (GO) molecular function annotation.

We refer to the embedding space learned by word2vec for a particular set of hyperparameters as $V_{e m b}$. We refer to the $k$ nearest neighbors of a domain $d$ as $C_{\text {nearest }}^{d}$ found using the Euclidean distance. To inspect the relative performance of $V_{e m b}$ on each of the following evaluations, we randomized all domain vectors and run each evaluation task. Tha is, irrespective of the embedding method parameters and only for each different space dimensionality we assigned to each domain vector a newly created random vector and save this random space to be evaluated.

\subsubsection{Domain hierarchy}

InterPro defines a strict family-subfamily relationship among domains. This relationship is based on sequence similarity of the domain signatures. We refer to the children of domain $p$ as $C^{p}$. We use these relationships to evaluate an embedding space, posing the following research question,

$R Q$ : Did vectors of hierarchically closely domains form clusters in the $V_{\text {emb }}$ ?

Evaluation We evaluate $V_{e m b}$ by retrieving $C_{\text {neart }}^{d}$ of each domain. For all learned embedding spaces, we measured their recall performance, Recall $_{\text {hier }}$ defined as follows:

$$
\text { Recall }_{\text {hier }}=\sum \frac{\left|C^{p} \cap C_{\text {nearest }}^{p}\right|}{\left|C^{p}\right|}
$$

\subsubsection{Secondary structure class}

We extracted the secondary structure of Interpro domains from the SCOPe database and form the following research question,

$R Q$ : Did vectors of domains, with same secondary structure class, form clusters in the $V_{\text {emb }}$ ?

Evaluation We evaluated $V_{e m b}$ by retrieving $C_{\text {nearest }}^{d}$ of each domain Then we applied stratified cross-fold validation and measured the performance of a $k$-nearest neighbor classifier to predict the structure clas of each domain. The intrinsic evaluation performance metric is the average accuracy across all folds, Accuracy ${ }_{S C O P e}$.

\subsubsection{Enzyme Commission (EC) primary class}

The enzymatic activity of each domain is given by its primary EC class (Fleischmann et al., 2004) and pose the following research question,

RQ: Did vectors of domains, with same secondary structure class, form clusters in the $V_{\text {emb }}$ ?

Evaluation We again evaluate $V_{e m b}$ using $k$ nearest neighbors in a stratified cross-validation setting. Average accuracy across all folds is again used to quantify the intrinsic quality of the embedding space.

\subsubsection{GO molecular function}

For our last intrinsic evaluation, we aimed to assess $V_{e m b}$ using the molecular function GO annotation. We extracted all molecular function GO annotations associated with each domain. ${ }^{1}$ Since model organisms have the most annotated proteins we created GO molecular function data sets for one example prokaryote (Escherichia coli denoted E.coli), one example simple eukaryote (Saccharomyces cerevisiae denoted

\footnotetext{
${ }^{1}$ In order to account for differences in specificity of different GO annotations, we always used the depth-1 ancestor of each annotation (that is, children of the root molecular function term, GO:0003674).
}

S.cerevisiae) and one complex eukaryote (Homo sapiens denoted Human) To assess our embeddings also for not highly annotated organisms, we included a molecular function data set for an example human pathogen (Plasmodium falciparum, denoted as Malaria). Finally, we pose the following research question

$R Q$ : Did vectors of domains, with same GO molecular function, form clusters in the $V_{\text {emb }}$ ?

Evaluation We again evaluate an embedding space using $k$ neares neighbors in a stratified cross-validation setting. Average accuracy across all folds is again used to quantify performance.

\subsection{Extrinsic evaluation}

In addition to assess the learned $V_{e m b}$ we also examine the performance change in downstream tasks. That is for three data sets-supervised tasks, we feeded the domain representations in simple neural networks and compare the performance of our model with state-of-the-art protein embeddings.

\subsubsection{Simple neural models for downstream task}

We consider a set of simple, well-established neural models to combine the domain embeddings for each protein to perform downstream tasks, that is, for extrinsic evaluation tasks. In particular, we use FastText (Joulin et al. 2017), convolutional neural networks (CNNs) (LeCun et al., 1998), and recurrent neural networks (RNNs) with long-short term memory (LSTM) cells (Hochreiter and Schmidhuber, 1997) and bi-directional LSTMs. We leave evaluation with more sophisticated models, such as transformers like BERT (Devlin et al., 2018), for future work.

The location data set is a multi-class task, thus we used as performance metric a multi-class generalization of area under the receiver operating characteristic curve (mc-AuROC). For the membrane data set which is a binary task, the performance metric was the area under the receiver operating characteristic curve (AuROC)

\subsubsection{TargetP}

We downloaded the TargetP data set provided by (Emanuelsson et al. 2000). To compare with ProtVecX we also used the non-plant data set. This data set consists of 2,738 proteins accompanying with their uniprot id, sequence and the cellular location label which can be nuclear, cytosol, pathway or signal and mitochondrial. Finally, we removed all instances with duplicate set of domains, resulting in total of 2,418 . This is a multiclass task and its class distribution is summarized in supplementary section E.

Evaluation For the TargetP we used the mc-AuROC performace metric.

\subsubsection{Toxin}

(Gacesa et al.,2016) introduced a data set associating protein sequence to toxic or other physiological content. To compare with ProtVecX we used the hard setting which provides uniprot id, sequence and the label toxin content or non-toxin content, for 15,496 proteins. Finally, we kept only the proteins with unique domain composition, resulting to 2,270 protein instances in total. This is a binary task and the class distribution is shown in supplementary section E.

Evaluation As Toxin data set is binary task, we used AuROC a performance metric.

\subsubsection{NEW}

We downloaded the NEW data set from (Li et al., 2017). For each of the 22,618 proteins the data set provides sequence and the EC number (class). The primary enzyme class, first digit of EC number, is our label on this prediction task, resulting in a multi-class task. Finally, we removed al instances with duplicate domain composition, resulting in a total of 14,434 
protein instances. The possible classes are 6 and the class distribution is shown in supplementary section $\mathrm{E}$.

Evaluation NEW data set is a multi-class task, thus we used mcAuROC as performance metric.

\subsubsection{Data partitioning}

We divided each data set into 70/30\% train and test splits. To perform model selection, we created inner three-fold cross validation sets on each train split.

Then we observed that the performance of classifier depending on protein domains is highly dependent on the out-of-vocabulary (OOV) domains, as first discussed in (Luong et al., 2015). OOV domains are all the domains contained in the test set, but not in the train. For TargetP, Toxin and NEW we observed that approximately $60 \%, 20 \%, 20 \%$ of test proteins contain at least one OOV domain. Thus, we run experiments based on this observation. For TargetP, containing the highest OOV, we split the test set into shorter sets by an increasing degree of OOV, namely $0 \%, 10 \%, 30 \%, 50 \%, 70 \%, 100 \%$. Then we learned models for the whole train set and benchmarked the performance on each of these test subsets.

For Toxin and NEW data set, experiencing low OOV, we seek to investigate the generalization of the produced classifier, by increasing the number of training examples that the model learn from and benchmark always in the entire test set. Thus, we created training splits of size, $10 \%$, $20 \%, 50 \%$ of the whole train set. To perform significance testing we created 10 random subsamples for each training split percentage.

\section{Results and discussion}

\subsection{Building domain architecture}

We downloaded the domain hits, protein2ipr.dat, for UniProt proteins from the InterPro version 75. This file contained 128660257 proteins with InterPro signature, making up the $80.9 \%$ of the total UniProtKB proteome (version 2019_06). For all these proteins we extracted the non-overlapping and non-redundant sequences, which we processed in the next section. The number of unique non-overlapping sequence was $(35183+1)$ plus the "GAP" domain and non-redundant was $(36872+1)$ plus the "GAP" Comparing to total number of domains in InterPro version 75 , which was 36872 , we observed that non-overlapping sequences captured $95.42 \%$ and the non-redundant captured $100 \%$ of the InterPro domains. Figure 2 demonstrates an example of constructed domain architectures by showing the domain architectures for the subdomains for the Tetrapyrrole methylase domain of the Diphthine synthase protein (see (Mitchell and et al., 2019) for more details)

\subsection{Training domain embeddings}

\subsubsection{Domain architecture}

Before we applied the word2vec package we examined the histograms of number of domains per protein for non-overlapping and non-redundant sequences. We show the histogram of the number of non-overlapping sequences per protein in Figure 3. We observed that these distributions are skewed and long-tailed. Then we used both CBOW and SKIP algorithms to learn domain embeddings. We used the following parameters sets. Based on the histograms we picked the window parameter for word to be 2 or 5 , $w=\{2,5\}$. For the number of dimensions, we used common values from the NLP literature, $\operatorname{dim}=\{50,100,200\}$. We trained the embeddings from 5 to 50 epochs with step size 5 epochs $e p=\{5,10,15, \ldots, 50\}$. Finally, all other parameters were set to their default values. For example, the negative sampling parameter was left to default, $n g=5$.

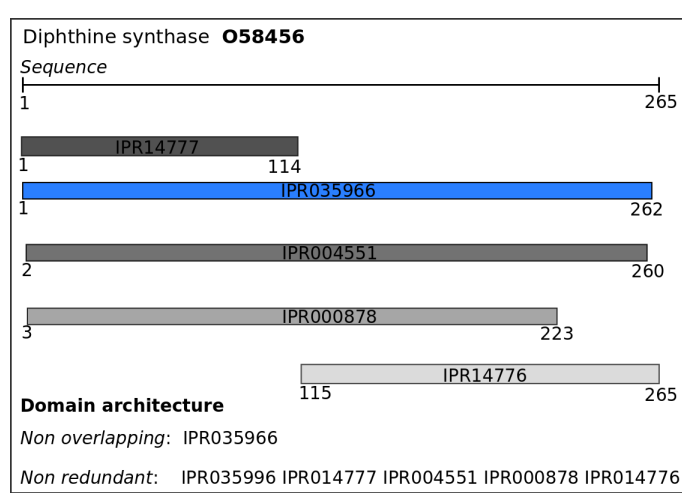

Fig. 2. Non overlapping and non-redundant domain architecture of Diphthine synthase protein. Because all domains are overlapping with the largest one, colored in blue, the nonoverlapping sequence is just the single longest domain (IPR035966). All other domain have a unique InterPro id, so the set of non-redundant sequences includes all presented domains sorted by starting position; we colored all these other domains in fading hues of gray based on their starting position.

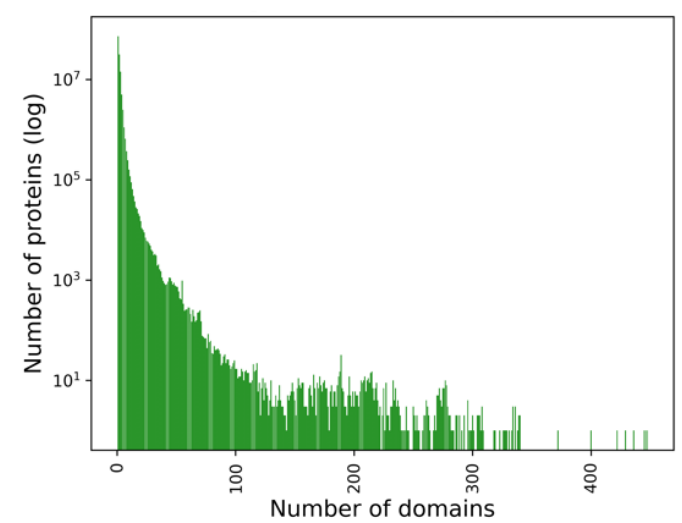

Fig. 3. Histogram of non-overlapping domains per protein, where the number of protein is in $\log _{10}$ scale.

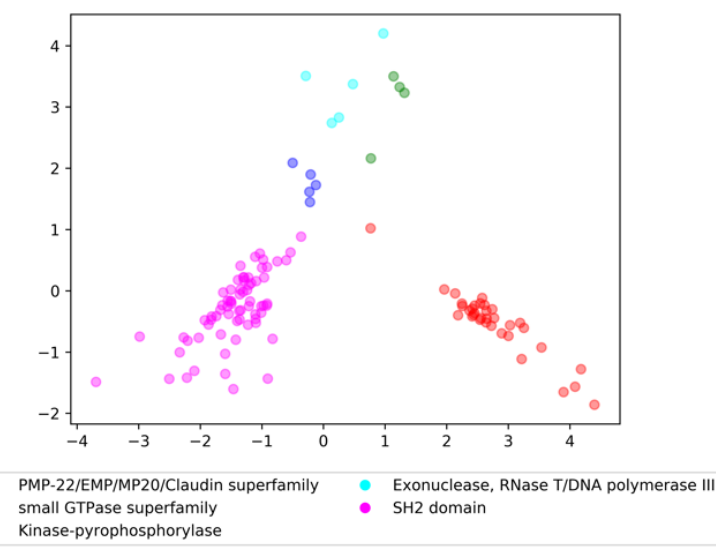

Fig. 4. Visualization of domain vectors for five domain superfamilies in best performing dom2vec space $\left(V_{e m b}^{\text {downstream }}\right)$

\subsection{Qualitative evaluation}

We randomly selected five InterPro domain superfamilies to perform the experiment. The selected domain superfamilies were $P M P$ 22/EMP/MP20/Claudin superfamily with parent InterPro id IPR004031, 
small GTPase superfamily with parent InterPro id IPR006689, Kinasepyrophosphorylase with parent InterPro id IPR005177, Exonuclease, RNase T/DNA polymerase III with parent InterPro id IPR013520 and SH2 domain with parent InterPro id IPR000980. Similarly to domain hierarchy, we loaded the parent-child tree $T_{h i e r}$, provided by InterPro, and for each domain superfamily starting from the parent domain we included recursively all domains that have subfamily relation with this parent domain. For example the Kinase-pyrophosphorylase domain superfamily has domain parent IPR005177, which in turn has two immediate domain subfamilies IPR026530, IPR026565 and IPR026565 has domain subfamily IPR017409, consequently the set of domains for Kinase-pyrophosphorylase domain superfamily is \{IPR005177,IPR026530,IPR026565,IPR017409\}.

We retrieved the vectors for each domain in a domain superfamily using the $V_{e m b}^{\text {downstream }}$, the best performing dom2vec space selected in Section 4.5. Finally, we visualized the two-dimensional PCA-reduced space at Figure 4. From this Figure 4, we observed that domains embeddings of each superfamily form well-separated clusters; the cluster of Exonиclease, RNase T/DNA polymerase III superfamily has the highest dispersion of all presented superfamilies.

\subsection{Novel intrinsic evaluation}

In the following, we evaluated each instance of learned embedding space $V_{e m b}$ for both non-overlapping and non-redundant representation of domain architectures. An instance of $V_{e m b}$ space is the embeddings space learned for each combination of the product non_overlap $\times w \times \operatorname{dim} \times e p$. Thus, the total number of embeddings spaces is $\mid$ non_overlap $|\times| w|\times|$ dim $|\times| e p \mid=2 \times 2 \times 3 \times 10=120$. Let $V_{e m b}^{i}$ denote such embedding space instance. In the following subsection we evaluated each $V_{e m b}^{i}$ instance for domain hierarchy, secondary structure, enzymatic process and GO molecular function.

\subsubsection{Domain hierarchy}

We loaded the parent-child tree $T_{h i e r}$, provided by InterPro, consisting of 2430 parent domains. Then for each $V_{e m b}^{i}$ we compared the actual and predicted children of each parent, and we averaged out the recall for all parents. For ease of presentation, we show only the results for nonredundant sequences at Table 1 and we provide the complete results in the Supplementary A. However, in the following we discuss the results.

From Tables S1 and 1 (appendix A), we observed that SKIP performed better overall, and the embeddings learned from non-redundant annotations always have better average recall values compared to nonoverlapping. The best performing $V_{e m b}^{i}$ achieved average Recall hier $_{\text {of }}$ 0.538 ; that embedding space was trained using non-redundant annotations with SKIP, $w=2$ and $\operatorname{dim}=200$. We plotted the histogram of recal values for this best performing space, Figure S1 (Suppl. A), and we observed that the embeddings space brought close domains with not known family-subfamily relation for almost the one third of the parent domain (827 out of 2430 ). To diagnose the reason for this moderate performance, we plotted the histogram of the number of children for each parent having recall 0, Figure S2 (Suppl. A). We observed that most of these parent have only one child. Consequently, the embedding space should have been very precise for each of these parent child relation in order to acquire better recall than 0 . We compared this moderate performance of $V_{e m b}$ with the performance of the randomized spaces, which was equal to 0 Thus, we concluded that our embedding spaces greatly outperformed each randomized space for domain hierarchy relation. Consequently, we can answer our initial question: the majority of domains of the same hierarchy are placed in close proximity in the embedding space.

\subsubsection{Secondary structure class}

We extracted the SCOPe class for each InterPro domain from the interpro.xml file. This resulted to 25196 domains with unknown secondary structure class, 9411 with single secondary structure class and 2265 domains with more than one assigned classes (multi-label). For clarity, we removed all multi-label and unknown instances leaving with 941 single-labeled instances; the class distribution of the resulting data set is shown in Table S2 (Supplementary B).

To answer the research question, we measured the performance of $C_{\text {nearest }}^{d}$ classifier in each $V_{e m b}^{i}$ to examine the homogeneity of the space with respect to the SCOPe class. We split the 9411 domains in 5-fold stratified cross validation sets. To test the change in prediction accuracy for an increasing number of neighbors, we used different sets of neighbors, namely, $k=\{2,5,20,40\}$. We summarized the results for the best performing $C_{\text {nearest }}^{d}$ which was for $k=2$ for non-redundant sequences in 2 . We show the respective table for non-overlapping sequences in the Suppl. B. We compared these accuracy measurements to the respective ones of the random spaces, and we found that the lowest accuracy values, achieved for CBOW $w=5$ using non-overlapping domains, are twice as high as the accuracy values of the random spaces for all possible dimensions. We observed again that the non-redundant domains resulted in higher accuracy compared to non-overlapping domains. The best performing $C_{\text {nearest }}^{d}$ was for 2-NN for (non-redundant, SKIP, $w=5, \operatorname{dim}=50, e p=25$ ) with average accuracy over the folds equal to 84.56. Consequently, we can answer our second research question: domain embeddings of the same secondary structure class form distinct clusters in a learned embedding space.

\subsubsection{Enzyme Commission (EC) primary class}

To this end we extracted the EC primary class from the interpro.xml file This resulted in 29354 domains with unknown EC, 7248 domains with only one EC and 721 with more than one EC. As before, we removed al multi-label and unknown instances leaving 7428 domains with known EC. For each $V_{e m b}^{i}$ we augmented a domain instance with its vector representation, and then we used $C_{\text {nearest }}^{d}$ to predict the EC. Please refer to the Supplementary C for the class distribution of EC task. We again omit the entire result tables and we discuss the main results.

We present the average Accuracy ${ }_{E C}$ obtained on embedding space learned using non-redundant sequences in Table 3 . We show the respective table for non-overlapping in the Suppl. C. We compared these accuracy measurements to the respective ones of the random spaces. We found that the minimum average $A_{c c u r a c y_{E C}}$ value was equal to 60.51 and wa achieved using CBOW $w=5$ for non-overlapping sequences. That value is approximately twice as large than the accuracy values of the random spaces for all possible dimensions (maximum average Accuracy $_{E C}$ fo random space with $d i m=100$ was 32.64 ). The best results were always produced using 2-NN, and we saw once more that SKIP trained on nonredundant sequences performed the best with maximum average accuracy of 90.85 (SKIP, $w=5, \operatorname{dim}=50, e p=30$ ). Consequently, we can answer our third research question: domain embeddings of the same EC primary class form distinct clusters in a learned embedding space.

\subsubsection{GO molecular function}

We parsed the GO annotation file of InterPro to extract first-level GO molecular function for domains for the four organisms. We followed the same methodology to examine the homogeneity of a $V_{e m b}$ with respect to GO molecular function annotations. Thus, for each $V_{e m b}^{i}$, we augmented each domain by its vector and its GO label, and we classified each domain using $C_{\text {nearest }}^{d}$. As before, we used 5-fold stratified cross-validation for evaluation. In our experiments, we varied the number of neighbors $k=\{2,5,20,40\}$ to test its influence to the change of performance. For space limitations, we summarized the performances showing only 


\begin{tabular}{llll}
\hline Emb. Meth, $w$ & dim $=50$ & dim $=100$ & dim=200 \\
\hline CBOW, $w=2$ & $0.406(e p=10)$ & $0.412(e p=10)$ & $0.414(e p=5)$ \\
CBOW, $w=5$ & $0.405(e p=30)$ & $0.402(e p=35)$ & $0.382(e p=10)$ \\
SKIP, $w=2$ & $0.512(e p=5)$ & $0.53(e p=5)$ & $\mathbf{0 . 5 3 8}(e p=5)$ \\
SKIP, $w=5$ & $0.507(e p=5)$ & $0.525(e p=5)$ & $0.524(e p=5)$ \\
random & 0 & 0 & 0 \\
Table 1. Average & Recall $l_{\text {hier }}$ & for non-redundant
\end{tabular}

sequences, best shown in bold case

\begin{tabular}{llll}
\hline Emb. Meth, $w$ & dim $=50$ & dim 100 & dim=200 \\
\hline CBOW, $w=2$ & $77.09(e p=5)$ & $76.35(e p=5)$ & $75.77(e p=5)$ \\
CBOW, $w=5$ & $78.15(e p=5)$ & $76.94(e p=5)$ & $76.84(e p=5)$ \\
SKIP, $w=2$ & $84.42(e p=45)$ & $84.42(e p=40)$ & $84.08(e p=30)$ \\
SKIP, $w=5$ & $\mathbf{8 4 . 5 6}(e p=25)$ & $84.06(e p=45)$ & $83.72(e p=10)$ \\
random & $23.39(k=40)$ & $23.49(k=40)$ & $22.76(k=20)$ \\
\hline Table 2. Average $C_{\text {nearest }}^{d}$ Accuracy ${ }_{S C O P e}$ for non-
\end{tabular}

redundant sequences $(k=2)$, best shown in bold case the best average accuracy over the number of neighbors. For the ease of presentation we omit the resulted tables for three first organisms and show only for Human; however, we discuss the results for all organisms. Please see supplementary D for full results.

For Malaria, the best average accuracy was 76.86 , scored by the 2-NN for the $V_{e m b}^{\text {malaria }}$ corresponding to (non-redundant, SKIP, $w=5, d i m=100, e p=40$ ). The minimum accuracy value was 56.94 and was achieved using CBOW with $w=5$ using non-overlapping sequences. We compared this minimum accuracy to the maximum accuracy obtained in a random space, which was 47.57 . That is, even the worst-performing dom2vec approach outperformed the random baseline by ten percent.

For E.coli, the best average accuracy was 81.72 , scored using 2 -NN for the $V_{e m b}^{\text {ecoli }}$ corresponding to (non-redundant,SKIP, $w=5$, dim=50, $e p=5$ ). The minimum accuracy value was 67.34 , achieved using CBOW with $w=5$ using non-overlapping sequences. For E.coli, the random baseline was still worse, with an accuracy of 64.46 .

For Yeast, the best average accuracy was 75.10 , scored using 5-NN for the $V_{e m b}^{\text {yeast }}$ corresponding to (non-redundant,SKIP, $w=5, \operatorname{dim}=50, e p=50$ ). The minimum accuracy value was 59.82, achieved using CBOW with $w=5$ using non-overlapping sequences. We compared this miminum accuracy to the maximum accuracy obtained in a random space, which was 53.73

For Human, the best average performance for non-redundant sequences are shown in Table 4 . The best average accuracy was 75.96 , scored by 2 -NN for the $V_{e m b}^{\text {human }}$ (non-redundant, SKIP, $w=5$,dim $=50, e p=40$ ). We compared the minimum accuracy values, obtained by CBOW with $w=5$, to accuracy values of the random spaces, and we found that the worstperforming dom $2 \mathrm{vec}$ was 20 percentage values higher than the random baseline.

For all four example organisms, we observed that the SKIP on non-redundant sequences produced $V_{e m b}$ where $C_{\text {nearest }}^{d}$ achieved the best average accuracy in. For the three out of the four organisms, the best performances were achieved for the lowest number of dimensions $(\operatorname{dim}=50)$. In all cases, we found that the worst-performing dom2vec embeddings outperformed the random baselines.

\subsection{Selecting trained embeddings}

Based on the previous four experiments, we aimed to evaluate the learned $V_{e m b}$ spaces and select the best domain embedding space for downstream tasks. In all experiments, the SKIP algorithm for non-redundant sequences created best performing embedding spaces. From the individual results, we saw that the configuration of parameters (non-redundant, SKIP, $w=5$, $d i m=50$ ) brought best results in $C_{\text {nearest }}^{d}$ performance for SCOPe, EC and GO for E.coli,Yeast,Human, second best for Malaria and the sixth best average recall (0.507) for the domain hierarchy relation. Therefore, for the next of the work $V_{e m b}^{\text {downstream }}$ denotes the space produced by (nonredundant, SKIP, $w=5$, dim $=50$ ).

\subsection{Extrinsic evaluation}

4.6.1 Extracting domain architecture

For each data set that contained the UniProt identifier for the protein instance, we extracted the domain architecture for non-redundant sequences, already created in Section 4.1. For all proteins whose UniProt identifier could not be matched, or for data sets not providing the protein identifier, we use InterProScan (Jones and et al., 2014) to find the domain hits per protein. For proteins without a domain hit after InterProScan, we created a protein-specific, artificial protein-long domain; for example, for the protein G5EBR8, we assigned a protein-long domain named "G5EBR8_unk_dom".

\subsubsection{Model selection}

To select which simple neural model we should compare to the baselines, we perform hyperparameter selection using an inner, three-fold cross validation on the training set; the test set is not used to select hyperparameters. We used common parameters, dropout of 0.5 , batch size of 64, the Adam optimizer (Kingma and Ba, 2015) with learning rate of 0.0003 , weight decay of 0 and number of epochs equal to 300 . As a final hyperparameter, we allowed updates to the learned domain embeddings, initialized by $V_{e m b}^{\text {downstream }}$. The results are shown in Supplementary E.

\subsection{Running baselines}

Then, we used the same network as the one in right side of Figure 5 of (Heinzinger et al., 2019); we refer to this network as SeqVecNet. Namely, the network first averages the 100 (ProtVec) or 1024 (SeqVec) dimensional embedding vector for a protein; it then applies a fully connected layer to compress a batch of such vectors into 32 dimensions. Next, a ReLU activation function (with 0.25 dropout) was applied to that vector, followed by batch normalization. Finally, another fully connected layer was followed by the prediction layer. As third baseline, we added the 1-hot of domains in order to investigate the performance change compared to dom2vec learned embeddings.

\subsubsection{Evaluation}

As discussed in 3.4.5, we performed two kinds of experiments. For TargetP, we sought to investigate the effect of OOV on the produced classifier compared to sequence-based embeddings classifiers, which do not experience OOV as their used sequence features are highly common in both train and test set. For Toxin and NEW datasets, we benchmarked the generalization of the produced classifier compared to the sequencebased embeddings classifiers. Finally, for both kinds of experiments, we used the trained models on each test set. Thus, this evaluation shows how differences in the training set affect performance on the test set. The produced performances are shown in Figure 5.

\subsubsection{Statistical significance}

For both Toxin and NEW, dom2vec significantly outperformed SeqVec, ProtVec and domains 1-hot vectors. In Toxin data set, we observed that 


\begin{tabular}{llll}
\hline Emb. Meth, $w$ & dim=50 & dim=100 & dim=200 \\
\hline CBOW, $w=2$ & $76.88(e p=5)$ & $75.85(e p=5)$ & $75.39(e p=5)$ \\
CBOW, $w=5$ & $80.89(e p=5)$ & $79.89(e p=5)$ & $77.16(e p=5)$ \\
SKIP, $w=2$ & $89.47(e p=35)$ & $89.06(e p=40)$ & $88.86(e p=5)$ \\
SKIP, $w=5$ & $\mathbf{9 0 . 8 5}(e p=30)$ & $90.41(e p=15)$ & $90.2(e p=5)$ \\
random & $33.62(k=40)$ & $32.06(k=40)$ & $32.28(k=40)$
\end{tabular}

Table 3. Average $C_{\text {nearest }}^{d}$ Accuracy $E C$ non-redundant sequences $(k=2)$, best shown in bold case

\begin{tabular}{llll}
\hline Emb. Meth, $w$ & dim=50 & dim=100 & dim=200 \\
\hline CBOW, $w=2$ & $66.94(e p=5)$ & $66.32(e p=5)$ & $66.32(e p=5)$ \\
CBOW, $w=5$ & $67.77(e p=5)$ & $65.87(e p=5)$ & $65.77(e p=5)$ \\
SKIP, $w=2$ & $74.77(e p=40)$ & $74.18(e p=5)$ & $73.14(e p=5)$ \\
SKIP, $w=5$ & $\mathbf{7 5 . 9 6}(e p=40)$ & $75.53(e p=10)$ & $74.98(e p=5)$ \\
random & $37.05(k=40)$ & $37.03(k=20)$ & $37.05(k=40)$
\end{tabular}

Table 4. Average $C_{\text {nearest }}^{d}$ Accuracy ${ }_{G O}$ for non-

redundant sequences,

when $k$ is not shown $k=2$, best shown in bold case
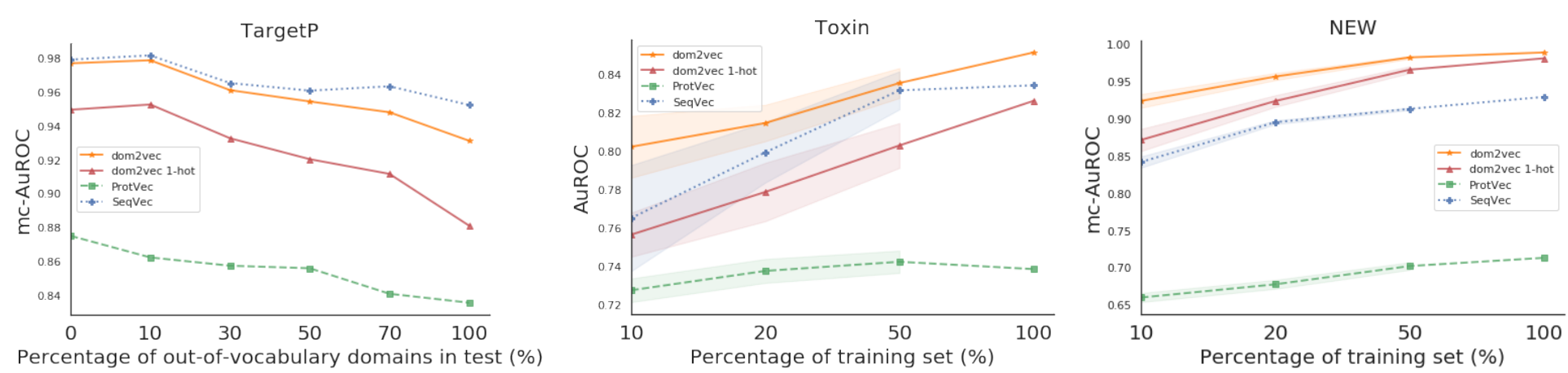

Fig. 5. TargetP, OOV experiment: learning in whole train and benchmark in test splits of increasing out-of-vocabulary degree. Toxin and NEW, generalization experiment: learning in increasing train splits, 10 replicates each, and benchmark in whole test sets. The marked points represent the mean performance on the test set and the shaded regions show one standard deviation above and below the mean.

ProtVec learned the less variant model, but with the trade-off obtaining the lowest performance (mc-AuROC). For NEW data set, the 1-hot representation was the second representation outperforming SeqVec and ProtVec allowing us to validate the finding that domain composition is the most important feature for enzymatic function prediction (Li et al., 2017)

For TargetP we validated our assumption that OOV will affect the performance of domains dependent classifiers. That is, for OOV in the range of $0-30 \%$ the dom $2 \mathrm{vec}$ classifier was comparable to the best performing model, SeqVec. However, when OOV increased more, then the performance of our model dropped, but still being competitive with the SeqVec. OOV is an known problem in NLP and as future work we would like to investigate ways to resolve this issue. Finally, dom2vec greatly outperformed the $1-$ hot representation, validating the NLP assumption that unsupervised embeddings improve classification on unseen words, in this context protein domains, compared to $1-$ hot word (domain) vectors.

\section{Conclusions}

We presented dom $2 \mathrm{vec}$, an approach for learning protein domain embeddings using the domain architectures deposed in InterPro.

We introduced a novel intrinsic evaluation based on the four source of biological knowledge of protein domains. We found that the dom $2 \mathrm{vec}$ vectors cluster by secondary structure, enzymatic function and GO molecular function however such representation could not capture domain hierarchy mostly for domain families of low cardinality. This result comes along with similar results in word embeddings, where it has been shown that, word vectors cluster by semantic and lexical similarity. This byproduct, allows us to draw an analog between words in natural languages and protein domains. Further this finding supports, in a data-driven way, the accepted modular evolution of proteins (Moore et al., 2008) and enhance the grammar of protein domain architectures (Yu et al., 2019) by giving insights on "forms" of collocations that can be found in such grammar.
In downstream task evaluation, dom2vec outperformed significantly domain 1 - hotvectors and state-of-the-art sequence-based embeddings for Toxin and NEW data sets. For the TargetP dom2vec was comparable to the best performing sequence-based embedding, Seqvec, for OOV up to $30 \%$. Thus we recommend to use dom 2 vec for prediction experiments when the OOV is limited, otherwise sequence-based embeddings can be used.

Finally, we hope that these embeddings will be used for prediction tasks, as well as, for creating data-driven hypothesis to augment our understanding of protein domain architectures.

\section{References}

Alley, E. C., Khimulya, G., Biswas, S., AlQuraishi, M., and Church, G. M. (2019). Unified rational protein engineering with sequence-based deep representation learning. Nature methods, 16(12), 1315-1322.

Almagro Armenteros, J. J., Sønderby, C. K., Sønderby, S. K., Nielsen, H., and Winther, O. (2017). DeepLoc: prediction of protein subcellular localization using deep learning. Bioinformatics, 33(21), 3387-3395.

Asgari, E. and Mofrad, M. R. K. (2015). Continuous distributed representation of biological sequences for deep proteomics and genomics. PLOS ONE, 10(11), e0141287.

Asgari, E., McHardy, A. C., and Mofrad, M. R. K. (2019). Probabilistic variablelength segmentation of protein sequences for discriminative motif discovery (DiMotif) and sequence embedding (ProtVecX). Scientific Reports, 9(3577).

Bepler, T. and Berger, B. (2019). Learning protein sequence embeddings using information from structure. In Proceedings of the $7^{\text {th }}$ International Conference on Learning Representations.

Chou, K.-C. and Cai, Y.-D. (2002). Using functional domain composition and support vector machines for prediction of protein subcellular location. Journal of Biological Chemistry, 277(48), 45765-45769.

Collobert, R. and Weston, J. (2008). A unified architecture for natural language processing: Deep neural networks with multitask learning. In Proceedings of the $25^{\text {th }}$ International Conference on Machine Learning.

Devlin, J., Chang, M.-W., Lee, K., and Toutanova, K. (2018). Bert: Pre-training of deep bidirectional transformers for language understanding. arXiv preprint arXiv:1810.04805.

Doğan, T., MacDougall, A., Saidi, R., Poggioli, D., Bateman, A., O’Donovan, C., and Martin, M. J. (2016). UniProt-DAAC: domain architecture alignment and classification, a new method for automatic functional annotation in UniProtKB. 
Bioinformatics, 32(15), 2264-2271

Drozd, A., Gladkova, A., and Matsuoka, S. (2016). Word embeddings, analogies, and machine learning: Beyond king-man+woman=queen. In Proceedings of the $26^{\text {th }}$ International Conference on Computational Linguistics: Technical Papers. $26^{t h}$ International Conference on Computational Linguistics: Technical Papers.
Eddy, S. R. (1998). Profile hidden markov models. Bioinformatics (Oxford, England), Eddy, S. R. (1998).
14(9), 755-763.

Emanuelsson, O., Nielsen, H., Brunak, S., and Von Heijne, G. (2000). Predicting subcellular localization of proteins based on their n-terminal amino acid sequence. Journal of Molecular Biology, 300(4), 1005-1016.

Fleischmann, A., Darsow, M., Degtyarenko, K., Fleischmann, W., Boyce, S., Axelsen, K. B., Bairoch, A., Schomburg, D., Tipton, K. F., and Apweiler, R. (2004). Intenz, the integrated relational enzyme database. Nucleic acids research, 32(suppl_1), D434-D437.

Forslund, K. and Sonnhammer, E. L. (2008). Predicting protein function from domain content. Bioinformatics, 24(15), 1681-1687.

Forslund, K. and Sonnhammer, E. L. (2012). Evolution of protein domain architectures. In Evolutionary Genomics, pages 187-216. Springer.

Fox, N. K., Brenner, S. E., and Chandonia, J.-M. (2013). SCOPe: Structura classification of proteins-extended, integrating SCOP and ASTRAL data and classification of new structures. Nucleic Acids Research, 42(D1), D304-D309.

Gacesa, R., Barlow, D. J., and Long, P. F. (2016). Machine learning can differentiate venom toxins from other proteins having non-toxic physiological functions. Peer Computer Science, 2, e90.

Gage, P. (1994). A new algorithm for data compression. The C Users Journal, 12(2), $23-38$

Heinzinger, M., Elnaggar, A., Wang, Y., Dallago, C., Nechaev, D., Matthes, F., and Rost, B. (2019). Modeling aspects of the language of life through transfer-learning protein sequences. BMC Bioinformatics, 20(723).

Hochreiter, S. and Schmidhuber, J. (1997) Long short-term memory. Neural Computation, 9(8), 1735-1780.

Jones, P. and et al. (2014). InterProScan 5: genome-scale protein function classification. Bioinformatics, 30(9), 1236-1240

Joulin, A., Grave, E., Bojanowski, P., and Mikolov, T. (2017). Bag of tricks fo efficient text classification. In Proceedings of the $15^{\text {th }}$ Conference of the European Chapter of the Association for Computational Linguistics.

Kingma, D. P. and Ba, J. L. (2015). Adam: A method for stochastic optimization. In Proceedings of the $3^{r d}$ International Conference on Learning Representations.

Lastra-Díaz, J. J., Goikoetxea, J., Taieb, M. A. H., García-Serrano, A., Aouicha, M. B., and Agirre, E. (2019). A reproducible survey on word embeddings and ontology-based methods for word similarity: linear combinations outperform the state of the art. Engineering Applications of Artificial Intelligence, 85, 645-665.

Le, Q. and Mikolov, T. (2014). Distributed representations of sentences and documents. In Proceedings of the $31^{\text {st }}$ International Conference on Machine Learning, .

LeCun, Y., Bottou, L., Bengio, Y., Haffner, P., et al. (1998). Gradient-based learning applied to document recognition. Proceedings of the IEEE, 86(11), 2278-2324. Li, Y., Wang, S., Umarov, R., Xie, B., Fan, M., Li, L., and Gao, X. (2017). DEEPre: sequence-based enzyme EC number prediction by deep learning. Bioinformatics, 34(5), 760-769.

Luong, T., Sutskever, I., Le, Q., Vinyals, O., and Zaremba, W. (2015). Addressing the rare word problem in neural machine translation. In Proceedings of the $53^{\text {rd }}$ Annual Meeting of the Association for Computational Linguistics.

Maaten, L. v. d. and Hinton, G. (2008). Visualizing data using t-sne. Journal of machine learning research, $\mathbf{9}(\mathrm{Nov}), 2579-2605$.

Marchler-Bauer, A., Bo, Y., Han, L., He, J., Lanczycki, C. J., Lu, S., Chitsaz, F. Derbyshire, M. K., Geer, R. C., Gonzales, N. R. et al. (2016). CDD/SPARCLE: functional classification of proteins via subfamily domain architectures. Nucleic Acids Research, 45(D1), D200-D203.

McInnes, L., Healy, J., and Melville, J. (2018). Umap: Uniform manifold approximation and projection for dimension reduction. arXiv preprin arXiv: 1802.03426

Mikolov, T., Sutskever, I., Chen, K., Corrado, G. S., and Dean, J. (2013a). Distributed representations of words and phrases and their compositionality. In Advances in Neural Information Processing Systems 26.

Mikolov, T., Chen, K., Corrado, G. S., and Dean, J. (2013b). Efficient estimation of word representations in vector space. In Proceedings of the $1^{\text {st }}$ International Conference on Learning Representations.

Mitchell, A. L. and et al. (2019). InterPro in 2019: improving coverage, classification and access to protein sequence annotations. Nucleic Acids Research, 47(D1), D351-D360.

Moore, A. D., Björklund, Å. K., Ekman, D., Bornberg-Bauer, E., and Elofsson, A. (2008). Arrangements in the modular evolution of proteins. Trends in Biochemical Sciences, 33(9), 444-451.

Pearson, K. (1901). Liii. on lines and planes of closest fit to systems of points in space. The London, Edinburgh, and Dublin Philosophical Magazine and Journal of Science, 2(11), 559-572.

Pennington, J., Socher, R., and Manning, C. (2014). GloVe: Global vectors for word representation. In Proceedings of the 2014 Conference on Empirical Methods in Natural Language Processing.

Peters, M. E., Neumann, M., Iyyer, M., Gardner, M., Clark, C., Lee, K., an Zettlemoyer, L. (2018). Deep contextualized word representations. In Proceeding of the $16^{\text {th }}$ Annual Conference of the North American Chapter of the Association for Computational Linguistics : Human Language Technologies.

Scaiewicz, A. and Levitt, M. (2015). The language of the protein universe. Current Opinion in Genetics \& Development, 35, 50-56.

Schnabel, T., Labutov, I., Mimno, D., and Joachims, T. (2015). Evaluation method for unsupervised word embeddings. In Proceedings of the 2015 Conference on Empirical Methods in Natural Language Processing.

Sonnhammer, E. L., Eddy, S. R., Birney, E., Bateman, A., and Durbin, R. (1998). Pfam: multiple sequence alignments and HMM-profiles of protein domains. Nucleic Acids Research, 26(1), 320-322.

Terrapon, N., Weiner, J., Grath, S., Moore, A. D., and Bornberg-Bauer, E. (2013). Rapid similarity search of proteins using alignments of domain arrangements. Bioinformatics, 30(2), 274-281.

The UniProt Consortium (2017). UniProt: the universal protein knowledgebase Nucleic Acids Research, 45(D1), D158-D169.

Woloszynek, S., Zhao, Z., Chen, J., and Rosen, G. L. (2019). 16s rRNA sequence embeddings: Meaningful numeric feature representations of nucleotide sequence that are convenient for downstream analyses. PLoS Computational Biology, 15(2), e1006721.

Yang, K. K., Wu, Z., Bedbrook, C. N., and Arnold, F. H. (2018). Learned protein embeddings for machine learning. Bioinformatics, 34(15), 2642-2648.

Yu, L., Tanwar, D. K., Penha, E. D. S., Wolf, Y. I., Koonin, E. V., and Basu, M. K. (2019). Grammar of protein domain architectures. Proceedings of the National Academy of Sciences, 116(9), 3636-3645. 


\section{Supplementary Information}

\section{Supplementary A: Domain hierarchy}

Average recall for all InterPro parents in $T_{h i e r}$, see main paper, for no overlapping sequences are shown in Tables S1. The histogram of average recall for best performing embedding space is shown at Figure S1. Finally, as a first diagnostic, the histogram of the number of children for each parent having Recall $_{\text {hier }}=0$, is shown at Figure S2

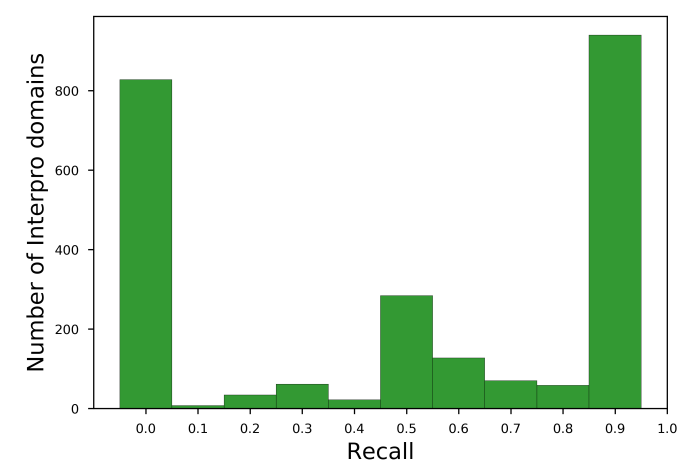

Fig. S1. Recall hier histogram for SKIP, $w=2, d i m=200, e p=5$ for nonredundant annotations.

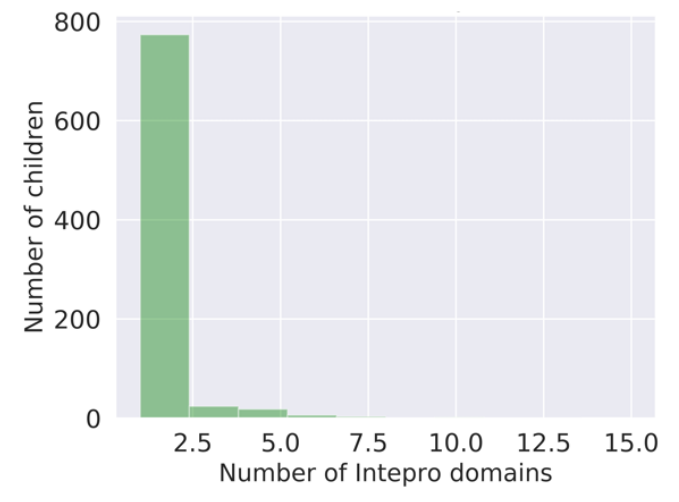

Fig. S2. Histogram of number of children for parents with Recall $_{h i e r}=0$.

\begin{tabular}{llll}
\hline Emb. Meth, $w$ & dim=50 & dim=100 & dim=200 \\
\hline CBOW, $w=2$ & $0.242(e p=15)$ & $0.259(e p=20)$ & $0.263(e p=15)$ \\
CBOW, $w=5$ & $0.242(e p=45)$ & $0.252(e p=30)$ & $0.25(e p=15)$ \\
SKIP, $w=2$ & $0.287(e p=20)$ & $\mathbf{0 . 3 1 6}(e p=30)$ & $0.32(e p=20)$ \\
SKIP, $w=5$ & $0.284(e p=20)$ & $0.302(e p=30)$ & $0.311(e p=30)$ \\
random & 0 & 0 & 0
\end{tabular}

Table S1. Average Recall hier $_{\text {for non-overlapping sequences, }}$ best shown in bold case

\section{Supplementary B: Secondary structure class}

Classes distribution of secondary structure class is shown at Table S2. Average $C_{\text {nearest }}^{d}$ Accuracy $_{S C O P e}$ for non-overlapping sequences shown in Tables S3.

\begin{tabular}{ll}
\hline SCOPe class & No. of domains \\
\hline a & 1,868 \\
$\mathrm{~b}$ & 1,806 \\
alb & 2,303 \\
a+b & 2,320 \\
multi-domain & 304 \\
membrane/cell & 309 \\
small & 501
\end{tabular}

Table S2. SCOPe class summary

\begin{tabular}{llll}
\hline Emb. Meth, $w$ & dim=50 & dim=100 & dim=200 \\
\hline CBOW, $w=2$ & $50.01(e p=30)$ & $50.69(e p=25)$ & $50.45(e p=20)$ \\
CBOW, $w=5$ & $49.59(e p=25)$ & $50.03(e p=25)$ & $48.82(e p=15)$ \\
SKIP, $w=2$ & $\mathbf{5 1 . 8 3}(e p=30)$ & $51.79(e p=20)$ & $51.78(e p=15)$ \\
SKIP, $w=5$ & $51.54(e p=35)$ & $51.65(e p=15)$ & $51.34(e p=15)$ \\
random & $22.75(k=40)$ & $24.18(k=40)$ & $23.39(k=40)$ \\
\hline $\begin{array}{llll}\text { Table S3. Average } C_{\text {nearest }}^{d} & \text { Accuracy } \\
\text { overlapping sequences }(k=2), \text { best shown in bold case }\end{array}$
\end{tabular}

Supplementary C: Enzyme Commission (EC) primary class

Classes distribution of EC primary class is shown at Table S4. Average $C_{\text {nearest }}^{d}$ Accuracy $E C$ for non-overlapping and non-redundant sequences shown in Tables S5 and 3 respectively.

\begin{tabular}{ll}
\hline EC primary class & No. of domain \\
\hline Oxidoreductases & 1102 \\
Transferases & 2490 \\
Hydrolases & 2190 \\
Lyases & 524 \\
Isomerases & 318 \\
Ligases & 448 \\
Translocases & 176 \\
\hline
\end{tabular}

Table S4. EC class summary

\begin{tabular}{llll}
\hline Emb. Meth, $w$ & $\operatorname{dim}=50$ & $\operatorname{dim}=100$ & $\operatorname{dim}=200$ \\
\hline CBOW, $w=2$ & $61.23(e p=10)$ & $61.33(e p=10)$ & $60.66(e p=15)$ \\
CBOW, $w=5$ & $61.22(e p=20)$ & $60.51(e p=10)$ & $60.61(e p=15)$ \\
SKIP, $w=2$ & $63.56(e p=10)$ & $\mathbf{6 3 . 9 2}(e p=20)$ & $62.58(e p=20)$ \\
SKIP, $w=5$ & $62.47(e p=10)$ & $63.44(e p=10)$ & $62.94(e p=15)$ \\
random & $31.51(k=40)$ & $32.64(k=40)$ & $31.68(k=20)$ \\
\hline Table S5. Average $C_{\text {nearest }}^{d}$ Accuracy $y_{E C}$ non-overlapping
\end{tabular}
sequences $(k=2)$, best shown in bold case 


\section{Supplementary D: GO molecular function}

\section{Malaria}

GO class distribution, $C_{\text {nearest }}^{d}$ Accuracy $G O$ for non-overlapping sequences and non-redundant sequences, for Malaria, are shown in S6, S7 and S8 respectively.

\section{E.coli}

GO class distribution, $C_{\text {nearest }}^{d}$ Accuracy $_{G O}$ for non-overlapping sequences and non-redundant sequences, for E.coli, are shown in S9, S10 and $\mathrm{S} 11$ respectively.

\section{Yeast}

GO class distribution, $C_{\text {nearest }}^{d}$ Accuracy $G O$ for non-overlapping sequences and non-redundant sequences, for Yeast, are shown in S12, S13 and S14 respectively.

\section{Human}

The $C_{\text {nearest }}^{d}$ Accuracy $_{G O}$ for non-overlapping sequences, for Human, is shown in Table S16.

\begin{tabular}{ll}
\hline GO class & No. of domains \\
\hline Catalytic activity & 676 \\
Binding & 440 \\
Structural molecule activity & 171 \\
Transporter activity & 63 \\
Molecular function regulator & 22 \\
Transcription regulator activity & 13 \\
Cargo receptor activity & 1 \\
Molecular carrier activity & 1 \\
\hline S6. GO molecular function distribution (Malaria)
\end{tabular}

Table S6. GO molecular function distribution (Malaria)
\begin{tabular}{llll}
\hline Emb. Meth, $w$ & $\operatorname{dim}=50$ & $\operatorname{dim}=100$ & $\operatorname{dim}=200$ \\
\hline CBOW, $w=2$ & $58.2(k=5, e p=35)$ & $58.24(k=5, e p=5)$ & $57.87(e p=5)$ \\
CBOW, $w=5$ & $57.87(e p=10)$ & $56.94(e p=10)$ & $57.1(e p=5)$ \\
SKIP, $w=2$ & $59.48(k=5, e p=10)$ & $59.82(e p=15)$ & $58.68(e p=10)$ \\
SKIP, $w=5$ & $\mathbf{6 0 . 6 1}(e p=10)$ & $59.01(e p=10)$ & $59.39(e p=5)$ \\
random & $46.21(k=40)$ & $46.62(k=40)$ & $45.99(k=40)$
\end{tabular}

Table S7. Average $C_{\text {nearest }}^{d}$ AccuracyGO for non-overlapping sequences, whenever $k$ is not shown $k=2$, best shown in bold case (Malaria)

\begin{tabular}{llll}
\hline Emb. Meth, $w$ & dim=50 & dim=100 & dim=200 \\
\hline CBOW, $w=2$ & $67.33(e p=5)$ & $64.88(e p=5)$ & $61.13(e p=5)$ \\
CBOW, $w=5$ & $66.74(e p=5)$ & $66.75(e p=5)$ & $63.89(e p=5)$ \\
SKIP, $w=2$ & $75.79(e p=35)$ & $75.64(e p=45)$ & $74.91(e p=5)$ \\
SKIP, $w=5$ & $76.79(e p=10)$ & $\mathbf{7 6 . 8 6}(e p=40)$ & $72.75(e p=20)$ \\
random & $46.58(k=40)$ & $46.22(k=40)$ & $47.57(k=40)$ \\
\hline Table S8. Average $C_{\text {nearest }}^{d}$ Accuracy A for non-redundant \\
sequences $(k=2)$, best shown in bold case (Malaria)
\end{tabular}

\begin{tabular}{|c|c|c|c|}
\hline \multicolumn{2}{|l|}{ GO class } & \multicolumn{2}{|c|}{ No. of domains } \\
\hline \multicolumn{2}{|c|}{ Catalytic activity } & \multicolumn{2}{|c|}{1565} \\
\hline \multicolumn{2}{|c|}{ Binding } & \multicolumn{2}{|c|}{476} \\
\hline \multicolumn{2}{|c|}{ Transporter activity } & \multicolumn{2}{|c|}{211} \\
\hline \multicolumn{2}{|c|}{ Structural molecule activity } & \multicolumn{2}{|c|}{117} \\
\hline \multicolumn{2}{|c|}{ Transcription regulator activity } & \multicolumn{2}{|c|}{39} \\
\hline \multicolumn{2}{|c|}{ Molecular function regulator } & \multicolumn{2}{|c|}{15} \\
\hline \multicolumn{2}{|c|}{ Molecular carrier activity } & \multicolumn{2}{|c|}{3} \\
\hline \multicolumn{2}{|c|}{ Translation regulator activity } & \multicolumn{2}{|c|}{1} \\
\hline \multicolumn{2}{|c|}{ Molecular transducer activity } & 1 & \\
\hline \multicolumn{4}{|c|}{$\begin{array}{lr}\text { Table S9. } & \text { GO } \\
\text { molecular function } & \text { fistribution (E.coli) }\end{array}$} \\
\hline Emb. Meth, $w$ & $\operatorname{dim}=50$ & $\operatorname{dim}=100$ & $\operatorname{dim}=200$ \\
\hline CBOW, $w=2$ & $67.66(k=5, e p=30)$ & $67.46(k=20, e p=5)$ & $67.34(k=20, e p=5)$ \\
\hline CBOW, $w=5$ & $67.78(k=5, e p=5)$ & $67.46(k=20, e p=5)$ & $67.34(k=20, e p=5)$ \\
\hline SKIP, $w=2$ & $68.15(k=5, e p=5)$ & $67.54(k=5, e p=5)$ & $67.75(k=20, e p=5)$ \\
\hline SKIP, $w=5$ & 69.1 $(k=5, e p=5)$ & $67.82(k=5, e p=5)$ & $68.15(k=5, e p=5)$ \\
\hline random & $64.46(k=40)$ & $64.46(k=40)$ & $64.46(k=40)$ \\
\hline
\end{tabular}

Table S10. Average $C_{\text {nearest }}^{d} A c c u r a c y_{G O}$ for non-overlapping sequences, best shown in bold case (E.coli)

\begin{tabular}{llll}
\hline Emb. Meth, $w$ & dim=50 & dim=100 & dim=200 \\
\hline CBOW, $w=2$ & $71.41(k=5, e p=5)$ & $68.45(k=5, e p=5)$ & $67.87(e p=5)$ \\
CBOW, $w=5$ & $74.95(e p=5)$ & $71.69(e p=5)$ & $68.91(e p=5)$ \\
SKIP, $w=2$ & $81.27(e p=5)$ & $80.32(e p=5)$ & $80.36(e p=5)$ \\
SKIP, $w=5$ & $\mathbf{8 1 . 7 2}(e p=5)$ & $81.64(e p=5)$ & $80.77(e p=5)$ \\
random & $64.38(k=40)$ & $64.46(k=40)$ & $64.38(k=40)$ \\
\hline
\end{tabular}

Table S11. Average $C_{\text {nearest }}^{d} A c c u r a c y_{G O}$ for non-redundant domains $(k=2)$, best shown in bold case (E.coli) 


\begin{tabular}{ll}
\hline GO class & No. of domains \\
\hline Catalytic activity & 1177 \\
Binding & 585 \\
Structural molecule activity & 208 \\
Transporter activity & 112 \\
Transcription regulator activity & 46 \\
Molecular function regulator & 40 \\
Translation regulator activity & 2 \\
Molecular transducer activity & 2 \\
Molecular carrier activity & 1 \\
Cargo adaptor activity & 1
\end{tabular}

\section{Supplementary E: Extrinsic evaluation}

Class distribution for TargetP, Toxin and NEW data sets shown in Tables S17, S18 and S19 respectively. Model selection over hyperparameters, including architecture, shown in Table S20.

Table S12. GO molecular function distribution (S.cerevisiae)

\begin{tabular}{llll}
\hline Emb. Meth, $w$ & dim=50 & dim=100 & dim=200 \\
\hline CBOW, $w=2$ & $60.05(k=20, e p=5)$ & $59.87(k=20, e p=5)$ & $59.87(k=20, e p=5)$ \\
CBOW, $w=5$ & $59.82(k=20, e p=15)$ & $60.24(k=20, e p=5)$ & $60.70(k=20, e p=5)$ \\
SKIP, $w=2$ & $60.74(k=5, e p=10)$ & $60.79(k=5, e p=10)$ & $61.53(k=5, e p=5$ \\
SKIP, $w=5$ & $\mathbf{6 1 . 3 8}(k=5, e p=10)$ & $60.75(k=20, e p=5)$ & $60.61(k=20, e p=$ \\
random & $53.36(k=40)$ & $53.64(k=40)$ & $53.64(k=40)$ \\
\hline Table S13. Average $C_{\text {nearest }}^{d}$ Accuracy ${ }_{G O}$ for non-overlapping \\
sequences, best shown in bold (S.cerevisiae $)$ & \\
& & & \\
\hline Emb. Meth, $w$ & dim=50 & dim=100 & dim=200 \\
\hline CBOW, $w=2$ & $64.37(k=5, e p=5)$ & $64.87(k=5, e p=5)$ & $62.4(k=5, e p=5)$ \\
CBOW, $w=5$ & $67.17(k=5, e p=50)$ & $65.11(k=5, e p=5)$ & $63.31(k=5, e p=5)$ \\
SKIP, $w=2$ & $73.36(k=5, e p=20)$ & $73.86(k=5, e p=5)$ & $72.29(k=5, e p=5)$ \\
SKIP, $w=5$ & $75.1(k=5, e p=50)$ & $74.1(k=5, e p=10)$ & $73.02(k=5, e p=5)$ \\
random & $53.59(k=40)$ & $53.73(k=40)$ & $53.18(k=40)$ \\
\hline
\end{tabular}

Table S14. Average $C_{\text {nearest }}^{d}$ AccuracyGO for non-redundant

sequences, best shown in bold (S.cerevisiae)

\begin{tabular}{ll}
\hline GO class & No. of domains \\
\hline Catalytic activity & 1,945 \\
Binding & 1,583 \\
Transporter activity & 377 \\
Molecular transducer activity & 355 \\
Structural molecule activity & 262 \\
Transcription regulator activity & 203 \\
Molecular function regulator & 168 \\
Cargo receptor activity & 9 \\
Molecular carrier activity & 1 \\
Cargo adaptor activity & 1 \\
\hline
\end{tabular}

Table S15. GO molecular function

distribution (Human)

\begin{tabular}{ll}
\hline Location & No. of proteins \\
\hline Nuclear & 1,072 \\
Cytosolic & 405 \\
Pathway/Signal & 605 \\
Mitochondrial & 304 \\
\hline Table S17. TargetP \\
\hline Toxicity & No. of proteins \\
\hline Toxin & 1,747 \\
Non toxin & 523 \\
\hline Table S18. Toxin & \\
\hline EC Primary class & No. of proteins \\
\hline Oxidoreductases & 2,234 \\
Transferases & 5,232 \\
Hydrolases & 4,099 \\
Lyases & 1124 \\
Isomerases & 731 \\
Ligases & 1,124 \\
\hline Table S19. NEW & \\
\hline
\end{tabular}

\begin{tabular}{llll}
\hline Emb. Meth, $w$ & dim=50 & dim=100 & dim=200 \\
\hline CBOW, $w=2$ & $57.7(e p=10)$ & $58.82(e p=10)$ & $58.04(k=5, e p=10)$ \\
CBOW, $w=5$ & $58.1(e p=30)$ & $58.9(e p=35)$ & $58.2(e p=10)$ \\
SKIP, $w=2$ & $60.51(k=5, e p=15)$ & $60.67(e p=10)$ & $59.3(e p=10)$ \\
SKIP, $w=5$ & $\mathbf{6 0 . 5 9}(k=5, e p=35)$ & $60.18(k=5, e p=10)$ & $59.84(k=5, e p=10)$ \\
random & $36.72(k=40)$ & $36.44(k=40)$ & $37.36(k=40)$ \\
\hline
\end{tabular}

Table S16. Average $C_{\text {nearest }}^{d} A_{c c u r a c y_{G O}}$ for non-overlapping sequences, when $k$ is not shown $k=2$, best shown in bold case (Human) 